\title{
Automated Decentralized Smart Sensor Network for Modal Analysis
}

\author{
S. H. Sim ${ }^{\text {a }}$, B. F. Spencer ${ }^{\text {a }}$, Jr., M. Zhang ${ }^{\mathrm{b}}$, and H. Xie ${ }^{\mathrm{c}}$ \\ ${ }^{a}$ Dept. of Civil and Environmental Engineering, University of Illinois at Urbana-Champaign, Urbana, \\ IL 61801, USA; \\ ${ }^{\mathrm{b}}$ Dept. of Civil Engineering, Shantou University, Shantou, Guangdong, China; \\ ${ }^{\mathrm{c} C o l l e g e ~ o f ~ C i v i l ~ E n g i n e e r i n g, ~ S h e n z h e n ~ U n i v e r s i t y, ~ S h e n z h e n, ~ G u a n g d o n g, ~ C h i n a ~}$
}

\begin{abstract}
Understanding the dynamic behavior of civil engineering structures is important to adequately resolve problems related to structural vibration. The dynamic properties of a structure are commonly obtained by conducting a modal survey that can be used for model updating, design verification, and improvement of serviceability. However, particularly for largescale civil structures, modal surveys using traditional wired sensor systems can be quite challenging to carry out due to difficulties in cabling, high equipment cost, and long setup time. Smart sensor networks (SSN) offer a unique opportunity to overcome such difficulties. Recent advances in sensor technology have realized low-cost smart sensors with on-board computation and wireless communication capabilities, making deployment of a dense array of sensors on large civil structures both feasible and economical. However, as opposed to wired sensor networks in which centralized data acquisition and processing are a common practice, the SSN requires decentralized algorithms due to the limitation associated with wireless communication; to date such algorithms are limited. This paper proposes a new decentralized hierarchical approach for modal analysis that reliably determines the global modal properties and can be implemented on a network of smart sensors. The efficacy of the proposed approach is demonstrated through several numerical examples.
\end{abstract}

Keywords: decentralized modal analysis, smart sensor, smart sensor network

\section{INTRODUCTION}

Research on structural vibration issues started in 1930s focusing on understanding the dynamic behavior of aircraft[1]; since that time, principles in structural dynamics have been widely considered in other applications. Identified dynamic characteristics are often used for model updating, design verification, and serviceability. A typical example is the retrofit of the London Millennium Footbridge to address unexpected large lateral vibrations caused by pedestrians[2]. To control bridge vibrations, fluid-viscous dampers and tuned mass dampers were installed on the bridge. In designing this damping system, understanding the dynamic characteristics of the as-built bridge was critical to ensuring efficient operation. The dynamic properties of structures are commonly obtained by conducting a modal survey that typically consists of measuring vibration signals of the structure and analyzing the measured data to estimate modal parameters such as natural frequencies, mode shapes, modal damping factors, and modal mass. However, for large-scale civil engineering structures, the long setup time, difficulties in cabling, and high equipment cost[3] makes conducting a modal survey using the conventional wired sensors challenging. For instance, imagine a modal survey of the Golden Gate Bridge. Miles of cables would be required to connect the central base station to the sensor nodes distributed along the towers and deck; installation would be both costly and time-consuming. Thus, more efficient means for modal surveys is desired particularly for large-scale civil structures.

Smart sensor networks (SSN) provides a promising alternative to traditional wired sensor networks. Recent advances in sensor technology have enabled low-cost smart sensors that have wireless communication, on-board processing, and multi-scale sensing capabilities; even lower prices are expected once smart sensors are in mass production. A variety of commercially available smart sensors has been developed in the last decade[4], opening a new era in structural health monitoring (SHM). Although some challenges, such as power consumption and long term reliability, still need to be resolved for the smart sensors to be more widely adopted, available smart sensors are already quite capable and can be expected to replace the traditional wired sensors for many applications. One particularly critical challenge relates to how the data is processed. Centralized data acquisition and processing schemes (See Fig. 1a) that are commonly used in traditional wired sensor systems cannot be employed in SSNs due to the limitation in wireless data communication 
speeds; bringing all data to a centralized location will result in severe data congestion in the SSN. This issue should be resolved for the SSN to be more widely deployed on large-scale civil structures.

A number of decentralized approaches have been proposed for conducting structural health monitoring and damage detection on networks of smart sensors. One of the decentralized approaches uses independent data processing, as shown in Fig. 1b[5][6][7][8][9]. Each sensor node processes measured data independently without communicating with other sensor nodes. The processed data, typically FFT or damage assessment results, is then sent to the base station. While the total amount of transferred data in the network is relatively small, important spatial information (e.g. mode shape) cannot be extracted. The decentralized approach proposed by Gao et al.[10] employs a coordinated computing strategy as shown in Fig. 1c, which has the ability to capture local spatial information. The sensor network in this scheme is divided into hierarchical communities, in which sensor nodes within each community communicate with each other in processing data; communication between communities is conducted through each community's manager node.

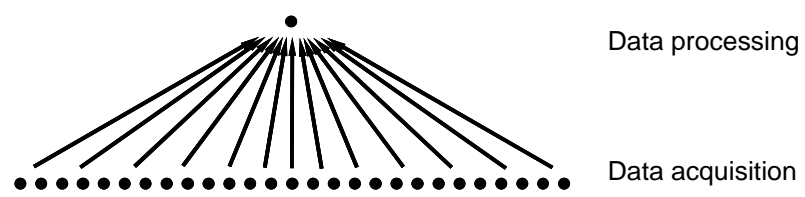

(a) Centralized data collection.

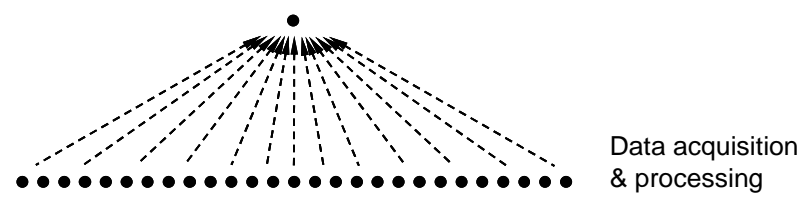

(b) Independent data processing.

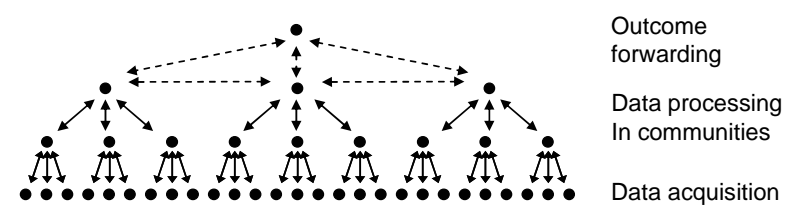

(c) Coordinated computing strategy.

Fig. 1. Data acquisition and processing schemes.

While a number of decentralized algorithms for structural health monitoring and damage detection have been published, relatively little effort has been devoted to developing such approaches for modal analysis. Zimmerman et al.[11] implemented a decentralized data processing scheme on a SSN to identify the vibration characteristics of the balcony in a historic theater in metropolitan Detroit. In the SSN, natural frequencies were determined at each node by the peak picking method[12][13] and sent to a central node where the final natural frequencies are decided. Based on the identified natural frequencies, phase differences between the responses of each pair of sensor nodes are determined in a sequential manner (see Fig. 2), and collected centrally to assemble the global mode shapes. While this decentralized algorithm was successfully implemented and tested, the approach requires a linear network topology and may result in substantial accumulation of errors in the global mode shapes.

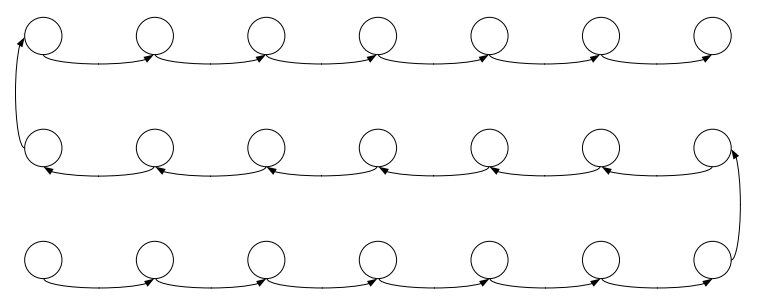

Fig. 2. Network topology by Zimmerman et al.[11]. 
This paper proposes an automated hierarchical decentralized approach for modal analysis that reliably determines the global modal properties based using a decentralized hierarchical network topology. The proposed method estimates local features within each local community; global modal properties are then calculated based on the collected local information. Herein, various sensor topologies for more accurate estimation of the global mode shape are considered. Numerical simulation of a plate and truss is provided to demonstrate the effectiveness of the proposed approach.

\section{PROBLEM FORMULATION}

The proposed approach consists of two parts: (i) local feature extraction and (ii) determination of global modal properties. In the SSN, local features are estimated in each local sensor community independently and subsequently collected at the base station, where the global modal properties are determined. This section describes this process.

\subsection{Local feature extraction}

Consider the structure and sensor network topology depicted in Fig. 3. The structure is divided into overlapping subdomains, represented by $\Omega_{i}(i=1, \cdots, n)$. Data aggregation and processing are conducted independently within each subdomain. In this study, two cases regarding the input excitation are considered. In the first, the input excitation is assumed to be measurable, allowing the transfer function to be estimated. By taking the inverse FFT of the transfer function, the impulse response function can be obtained and used as input to the Eigensystem Realization Algorithm (ERA)[14]. The second case assumes that the input excitation is unavailable, and the Natural Excitation Technique (NExT)[15] is employed. In this approach, the cross-correlation functions between the measured responses are used as the input to ERA. In both cases, only the identified local information is collected centrally for determination of the global modal properties. More information regarding implementation of these two approaches on smart sensor networks can be found in Nagayama and Spencer[16].

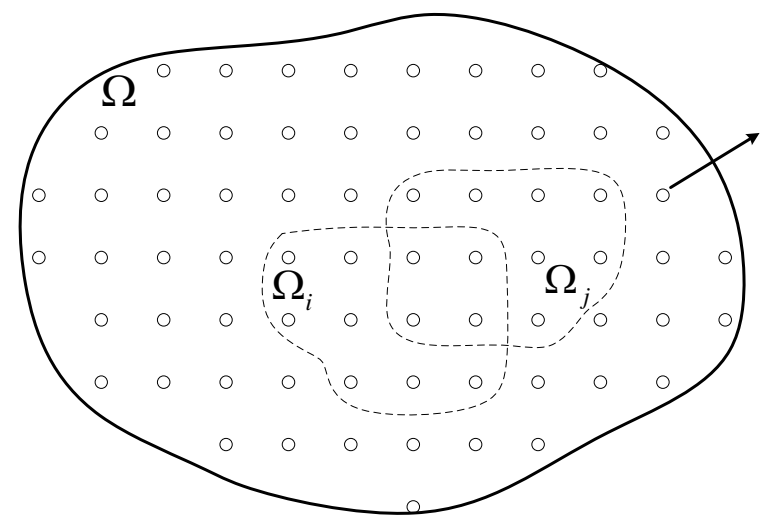

Fig. 3. Structure and overlapping subdomains $(i, j=1 \sim n)$.

\subsection{Determination of global modal properties}

Once the local information is collected centrally, the first task is to delineate the true modes from the noise modes. In this study, the true modes are selected based on the number of identified natural frequencies from the subdomains[11]. The true modes should be identified in the majority subdomains, while the noise modes will randomly appear in the subdomains. Thus, if a specific natural frequency is identified in a substantial number of the subdomains, it is considered as a true mode. If ERA fails to find the true mode in certain subdomains, the cross spectrum is alternatively used to estimate the local mode shapes. Once the true modes are determined, the corresponding mode shapes can be combined together; the remainder of this section describes this process.

Consider global mode shape $\phi_{\Omega}^{m}$ for the $m^{\text {th }}$ mode, along with the previously determined local mode shapes $\phi_{\Omega_{1}}^{m}, \phi_{\Omega_{2}}^{m}, \cdots, \phi_{\Omega_{n}}^{m}$ associated with respective subdomains. The local mode shapes $\phi_{\Omega_{i}}^{m}$ and $\phi_{\Omega_{j}}^{m}$ associated with two neighboring subdomains can be expressed as 


$$
\phi_{\Omega_{i}}^{m}=\left(\begin{array}{c}
1 \\
\phi_{i, 2} \\
\vdots \\
\phi_{i, p} \\
\phi_{i, 1}^{o} \\
\vdots \\
\phi_{i, r}^{o}
\end{array}\right) \quad \text { and } \quad \phi_{\Omega_{j}}^{m}=\left(\begin{array}{c}
1 \\
\phi_{j, 2} \\
\vdots \\
\phi_{j, q} \\
\phi_{j, 1}^{o} \\
\vdots \\
\phi_{j, r}^{o}
\end{array}\right)
$$

where the superscript $o$ denotes the overlapping node in the $i^{\text {th }}$ and $j^{\text {th }}$ subdomains, $r$ is the number of the overlapping nodes, and $p$ and $q$ are the number of non-overlapping nodes in the $i^{\text {th }}$ and $j^{\text {th }}$ subdomains, respectively. To allow assembly, the mode shapes in Equation (1) should be rescaled to have the same values at the overlapping nodes, i.e.,

$$
R_{i}\left(\begin{array}{c}
\phi_{i, 1}^{o} \\
\vdots \\
\phi_{i, r}^{o}
\end{array}\right)=R_{j}\left(\begin{array}{c}
\phi_{j, 1}^{o} \\
\vdots \\
\phi_{j, r}^{o}
\end{array}\right)
$$

where $R_{i}$ and $R_{j}$ are the normalization factors for the mode shapes $\phi_{\Omega_{i}}^{m}$ and $\phi_{\Omega_{j}}^{m}$, respectively. The global mode shape is the union of the local mode shapes as

$$
\phi_{\Omega}^{m}=\bigcup_{i=1}^{n} R_{i} \phi_{\Omega_{i}}^{m}
$$

In the presence of noise, the solution to Equation (2) for any $r>1$ does not exist in general. Therefore, the normalization factor $R_{i}(i=1,2, \cdots, n)$ must be approximately determined, for example as a solution in the leastsquare sense. Because the subdomains are interconnected, Equation (2) can be expanded up to $n(n-1) / 2$ equations for all pairs of the overlapping local mode shapes as follows.

$$
\begin{gathered}
\phi_{\Omega_{1} \Omega_{2}}^{m}=R_{2} \phi_{\Omega_{2} \Omega_{1}}^{m}+\varepsilon_{12} \\
\phi_{\Omega_{1} \Omega_{3}}^{m}=R_{2} \phi_{\Omega_{3} \Omega_{1}}^{m}+\varepsilon_{13} \\
\vdots \\
\phi_{\Omega_{1} \Omega_{n}}^{m}=R_{2} \phi_{\Omega_{n} \Omega_{1}}^{m}+\varepsilon_{1 n} \\
R_{2} \phi_{\Omega_{2} \Omega_{3}}^{m}=R_{3} \phi_{\Omega_{2} \Omega_{3}}^{m}+\varepsilon_{23} \\
\vdots \\
R_{2} \phi_{\Omega_{2} \Omega_{n}}^{m}=R_{3} \phi_{\Omega_{n} \Omega_{2}}^{m}+\varepsilon_{2 n} \\
\vdots \\
R_{n-1} \phi_{\Omega_{n-1} \Omega_{n}}^{m}=R_{n} \phi_{\Omega_{n} \Omega_{n-1}}^{m}+\varepsilon_{(n-1) n}
\end{gathered}
$$

where $\phi_{\Omega_{i} \Omega_{j}}^{m}$ is the $m^{\text {th }}$ local mode shape in the $i^{\text {th }}$ domain at the nodes that overlap the $j^{\text {th }}$ domain, and $\varepsilon_{i j}$ is the error between the mode shapes. Note that the normalization factor for the first subdomain $R_{1}$ is selected to be 1 . In matrix form, Equation (4) becomes

$$
y=X R+\varepsilon
$$

where 


$$
\boldsymbol{y}=\left(\begin{array}{c}
\phi_{\Omega_{1} \Omega_{2}}^{m} \\
\phi_{\Omega_{1} \Omega_{3}}^{m} \\
\vdots \\
\phi_{\Omega_{1} \Omega_{n}}^{m} \\
0 \\
\vdots \\
0
\end{array}\right), \boldsymbol{X}=\left(\begin{array}{cccc}
\phi_{\Omega_{2} \Omega_{1}}^{m} & 0 & \cdots & 0 \\
0 & \phi_{\Omega_{3} \Omega_{1}}^{m} & \cdots & 0 \\
\vdots & \vdots & \ddots & \vdots \\
0 & 0 & \cdots & \phi_{\Omega_{n} \Omega_{1}}^{m} \\
-\phi_{\Omega_{2} \Omega_{3}}^{m} & \phi_{\Omega_{3} \Omega_{2}}^{m} & \cdots & 0 \\
\vdots & \vdots & \ddots & \vdots \\
-\phi_{\Omega_{2} \Omega_{n}}^{m} & 0 & \cdots & \phi_{\Omega_{n} \Omega_{2}}^{m} \\
\vdots & \vdots & \vdots & \vdots \\
-\phi_{\Omega_{n-1} \Omega_{n}}^{m} & 0 & \cdots & \phi_{\Omega_{n} \Omega_{n-1}}^{m}
\end{array}\right), \boldsymbol{R}=\left(\begin{array}{c}
R_{2} \\
R_{3} \\
\vdots \\
R_{4}
\end{array}\right) \text {, and } \boldsymbol{\varepsilon}=\left(\begin{array}{c}
\varepsilon_{12} \\
\varepsilon_{13} \\
\vdots \\
\varepsilon_{1 n} \\
\varepsilon_{23} \\
\vdots \\
\varepsilon_{2 n} \\
\vdots \\
\varepsilon_{(n-1) n}
\end{array}\right)
$$

The estimator $\hat{\boldsymbol{R}}$ that minimizes the square of errors $\boldsymbol{\varepsilon}^{T} \boldsymbol{\varepsilon}$ is given by[17]

$$
\hat{\boldsymbol{R}}=\left(\boldsymbol{X}^{T} \boldsymbol{X}\right)^{-1} \boldsymbol{X}^{T} \boldsymbol{y}
$$

Using the normalization factor $\boldsymbol{R}$, the local mode shapes are scaled and assembled to obtain the global mode shape. At the overlapping nodes, the local mode shapes are averaged to obtain the associated values of the global mode shape.

The accuracy of the combined global mode shapes can be evaluated by the error between the combined and reference global mode shapes, $\phi_{\Omega}^{m}$ and $\phi_{\Omega, \text { ref }}^{m}$, respectively:

$$
e_{m}=\phi_{\Omega}^{m}-\phi_{\Omega, r e f}^{m}
$$

The reference global mode shapes are estimated using all measured accelerations simultaneously. Note that the reference global mode shapes correspond to the centralized data acquisition and processing scheme commonly used in the wired sensor networks, while the proposed decentralized approach is for the SSN. For the error $e_{m}$ to be meaningful, the two global mode shapes should be appropriately normalized. Thus, $\phi_{\Omega, \text { ref }}^{m}$ is normalized so that the largest element in $\phi_{\Omega, \text { ref }}^{m}$ is equal to 1 , and $\phi_{\Omega}^{m}$ is then scaled to minimize the square error between $\phi_{\Omega}^{m}$ and $\phi_{\Omega, \text { ref }}^{m}$ :

$$
\min \left(\phi_{\Omega}^{m}-\phi_{\Omega, \text { ref }}^{m}\right)^{T}\left(\phi_{\Omega}^{m}-\phi_{\Omega, \text { ref }}^{m}\right)
$$

Once normalized, the error $e_{m}$ of the combined global mode shapes can be calculated.

\section{NUMERICAL EXAMPLE}

A numerical example of a plate is provided to validate the proposed approach. In this example, the effects of the sensor topologies on the accuracy of the combined global mode shapes are investigated.

\subsection{Numerical model}

Consider the uniform plate shown in Fig. 4. The top and left edges of the plate are considered as fixed, and the bottom and right edges are simply supported. The plate is assumed to be $0.7 \mathrm{~m}$ by $1 \mathrm{~m}$ and made of steel having an elastic modulus of $200 \mathrm{GPa}$, a Poisson ratio of 0.3 , mass density of $7.83 \times 103 \mathrm{~kg} / \mathrm{m} 3$, and thickness of $1 \mathrm{~mm}$. The numerical model of the plate is implemented in MATLAB using the 12 DOF (3DOF at each node) rectangular Kirchhoff plate element known as the ACM element[18]. The shape functions are selected to be incomplete, $4^{\text {th }}$-order polynomials in the $x$ - and $y$-directions with 12 terms. The first seven out-of-plane mode shapes of the plate model are presented in Fig. 5 , and the transfer functions between the input excitation applied vertically at the nodes marked by an " $\mathrm{X}$ " and the accelerations at nodes N1 and N2 (see Fig. 4) are shown in Fig. 6.

A total of 63 evenly spaced sensor nodes are selected as shown in Fig. 4 to obtain vertical accelerations under the input excitation. As summarized in Table 2, four different simulation cases are considered: an impulse loading (cases 1 and 2) or a band-limited white noise up to $50 \mathrm{~Hz}$ (cases 3 and 4). 5\% RMS noise is added to all measurements. ERA is used to identify the mode shapes and frequencies in cases 1,2 , and 3, whereas NExT/ERA is used in case 4 . As the input 
excitation to large-scale civil engineering structures is not generally available, output-only modal analysis for random excitations is often employed; thus, case 4 may be considered as the most important for structural health monitoring.

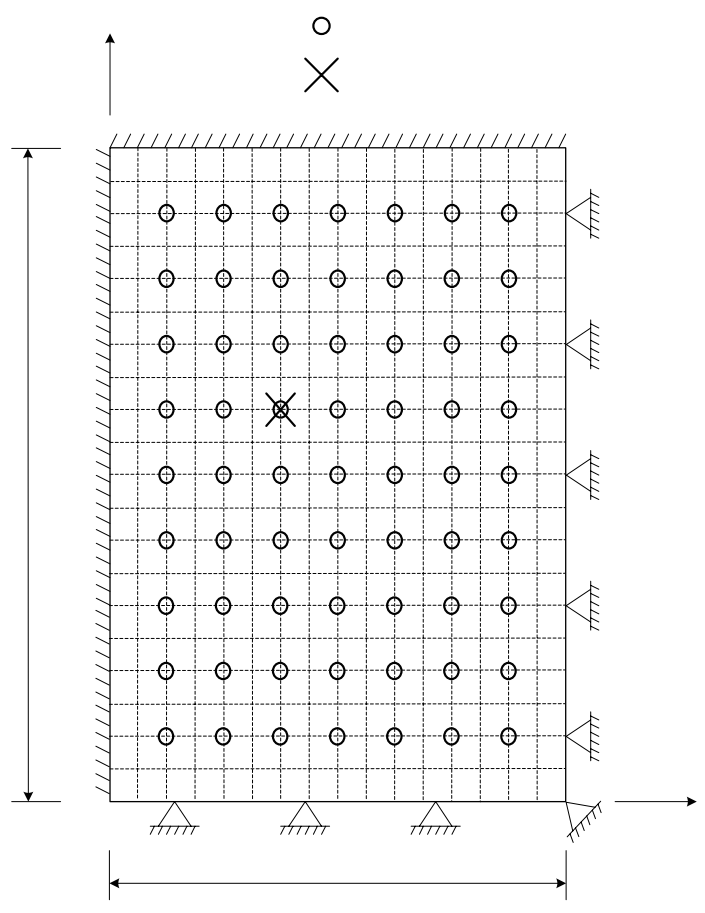

Fig. 4. Plate model.

$f_{n}=10.1554 \mathrm{~Hz}$

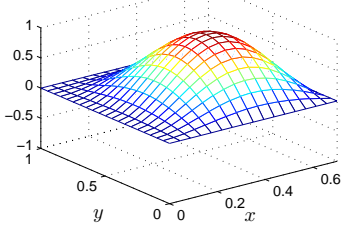

$f_{n}=30.7159 \mathrm{~Hz}$

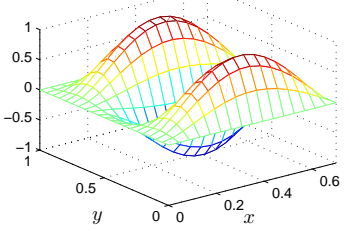

$f_{n}=48.479 \mathrm{~Hz}$

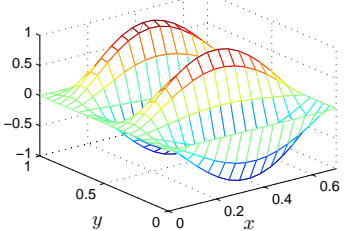

$f_{n}=17.8777 \mathrm{~Hz}$

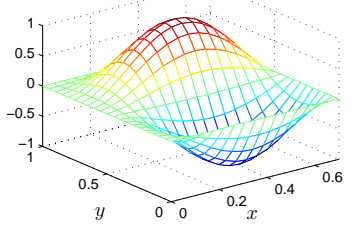

$f_{n}=34.3809 \mathrm{~Hz}$
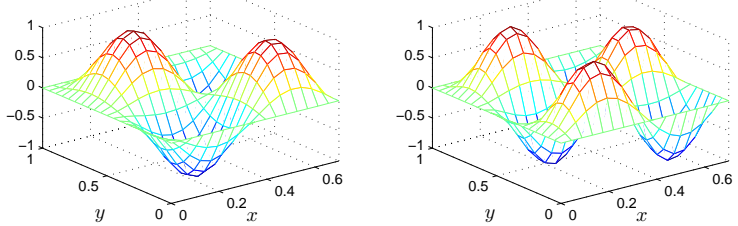

Fig. 5. Global mode shapes from the Finite Element model. 


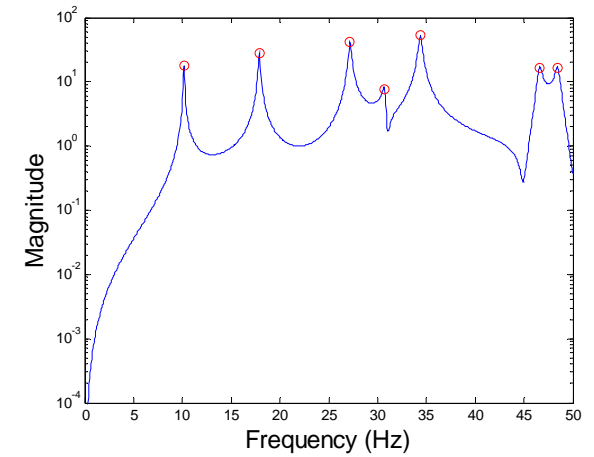

(a) $\mathrm{N} 1$

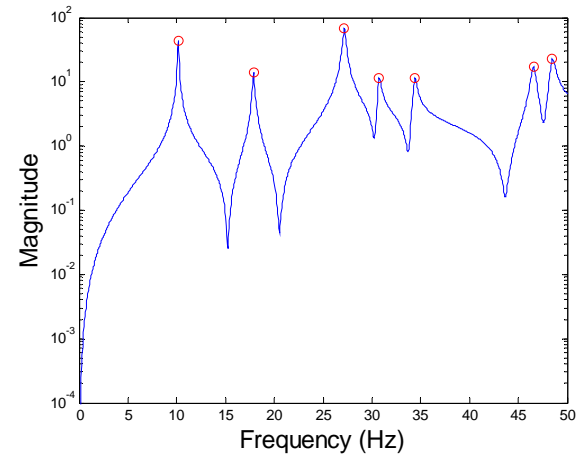

(b) $\mathrm{N} 2$

Fig. 6. Transfer functions (Circles represent natural frequencies).

Table 1. Global and local mode shapes.

\begin{tabular}{|c|c|c|}
\hline Excitation type & Input measured & Input not measured \\
\hline Impulse & Case 1 (ERA) & Case 2 (ERA) \\
\hline Band-limited white noise & Case 3 (ERA) & Case 4 (NExT/ERA) \\
\hline
\end{tabular}

\subsection{Sensor topology}

To investigate the effect of the sensor topologies on the combined global mode shapes, the nodes are grouped in four different ways, as shown in Fig. 7: (a) Topology 1: two nodes in each group with one overlapping node, (b) Topology 2: four nodes in each group with two overlapping nodes, and (c) Topology 3: nine nodes in each group with three overlapping nodes. Note that Topology 1 corresponds to the approach adopted by Zimmerman et al.[11].

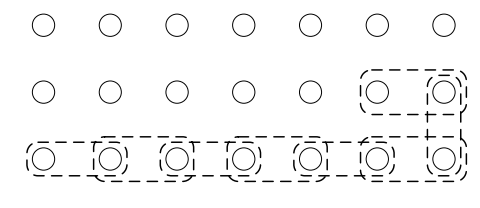

(a) Topology 1: 2 nodes in each group.

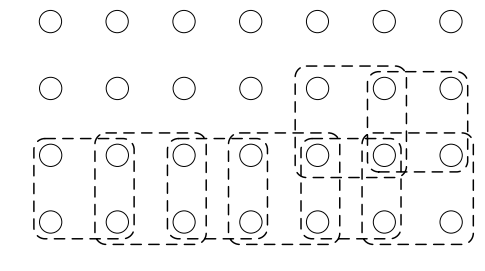

(b) Topology 2: 4 nodes in each group.

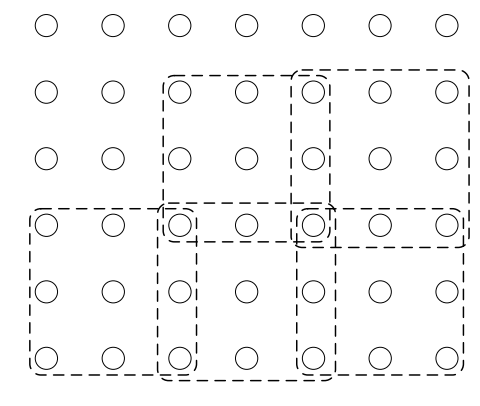

(c) Topology 3: 9 nodes in each group.

Fig. 7. Schematic view of subdomains (dashed line).

From the data communication perspective, having fewer local groups is advantageous due to less amount of data being transmitted over the radio. In a local group, the reference sensor sends sensor data that is generally long, while all other sensors transmits the condensed data such as the correlation function of the cross spectrum. Thus, the amount of transmitted data can be reduced by adopting a topology with less number of local groups. Assuming the time history record of length $N$ and $n_{d}$ times averaging without any overlap between spectral windows, the total number of transmitted data points[16] is at most

$$
N_{\text {data }}=n_{g}\left(N \cdot n_{d}+\frac{N}{2} \cdot\left(n_{s}-1\right)\right)
$$

where $n_{g}$ and $n_{s}$ are the numbers of local groups and sensor nodes, respectively. From Equation (9) with $N=1024$, $N_{\text {data }}$ are about $1.30 \times 10^{6}, 1.06 \times 10^{6}$, and $0.29 \times 10^{6}$ for topology 1 , 2, and 3, respectively. Thus, Topology 3 has the least data communication requirement. 


\subsection{Determination of true modes}

As previously described, the number of groups in which a natural frequency is locally identified is utilized to delineate between true modes and noise modes. The natural frequencies estimated in each group are collected, and if the number of collected natural frequencies in a specific frequency range is greater than a predetermined threshold value, it is assumed to be a true mode. The threshold is selected to be $70 \%$ of the number of local groups. The frequency range in which identified frequencies are counted is $\left(f_{c}-\Delta f \quad f_{c}+\Delta f\right)$ where $f_{c}$ is the central frequency of the range and $2 \Delta f$ is the width. In this study, $f_{c}$ and $\Delta f$ are selected as follows.

$$
\begin{aligned}
& f_{c}=r \cdot \frac{f_{s}}{N_{F F T}} \quad r=1,2, \cdots \\
& \Delta f=2 \cdot \frac{f_{s}}{N_{F F T}}
\end{aligned}
$$

where $f_{s}$ is the sampling rate, $N_{F F T}$ is the number of FFT points, and $r$ is any positive integer such that $f_{c}$ is less than the bandwidth of the input excitation. Note that the adjacent ranges are made to overlap with each other to prevent the case that the identified frequencies are evenly distributed over two adjacent non-overlapping ranges.

Fig. 8 shows the typical number of groups in which a natural frequency is found in case 4 (random excitation, input not measured). The identified frequencies in the local groups are concentrated at several frequencies such as about $10 \mathrm{~Hz}$, $18 \mathrm{~Hz}, 27 \mathrm{~Hz}, 31 \mathrm{~Hz}, 34 \mathrm{~Hz}, 47 \mathrm{~Hz}$, and $49 \mathrm{~Hz}$ that are finally considered as corresponding to true modes. In some frequency ranges, the number of local groups is greater than the total number of local groups due to noise modes that are closely located to the true modes. In this case, the frequency that is closest to $f_{c}$ is considered as the natural frequency of the local group.

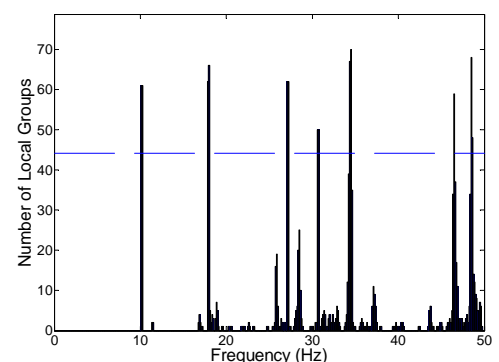

(a) Topology 1

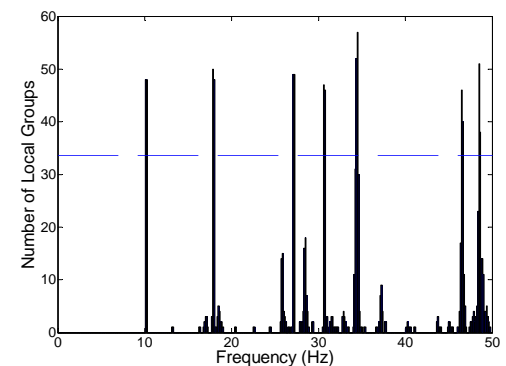

(b) Topology 2

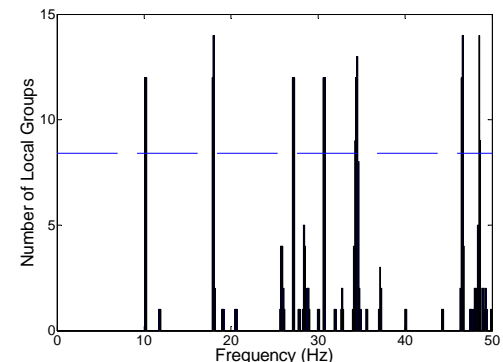

(c) Topology 3

Fig. 8. Number of identified natural frequencies (case 4: random excitation and input not measured).

\subsection{Global mode shapes}

The global mode shapes can be assembled with the local mode shapes using the proposed method described in section 2.2. If a true mode is not identified in a certain local group, cross spectra of the accelerations in the group are alternatively utilized to estimate the local mode shapes. The cross spectrum values at the spectral line nearest to the natural frequency of the majority groups are assumed as the mode shape. Note that mode shapes obtained only from the cross spectra may not be as accurate as those from the ERA because the cross spectra have values only at the spectral lines.

The error $e_{j}$ defined in Equation (7) is calculated to assess the accuracy of the combined global mode shape. Typical plots of the error $e_{2}$ for each topology in case 4 are shown in Fig. 9. Improvement in accuracy is clearly shown for Topologies 2 and 3, where a larger size of local groups and more overlapping nodes are employed.

Two error measures are considered to quantitatively evaluate the accuracy of the combined global mode shapes. The first is the maximum error for the $j^{\text {th }}$ mode:

$$
E_{\text {max }, j}=\max \left(\left|e_{j, 1}\right|,\left|e_{j, 2}\right|, \cdots,\left|e_{j, n}\right|\right)
$$


where $\mathrm{n}$ is the number of elements in $e_{j}$, and $e_{j, i}$ are the $i^{\text {th }}$ element of the error $e_{j}$. The second error measure is the average error for the $j^{\text {th }}$ mode:

$$
E_{\text {avg }, j}=\operatorname{mean}\left(\left|e_{j, 1}\right|,\left|e_{j, 2}\right|, \cdots,\left|e_{j, n}\right|\right)
$$

When a mode is not identified, the error of the mode is set to $100 \%$.

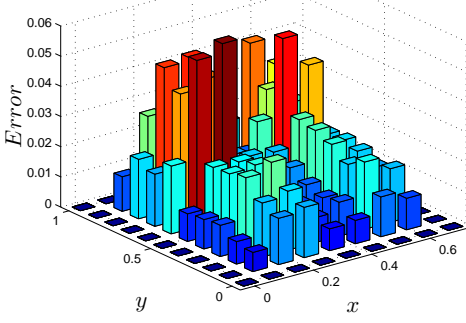

(a) Topology 1

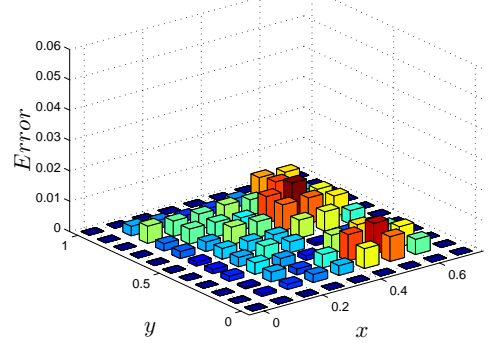

(b) Topology 2.

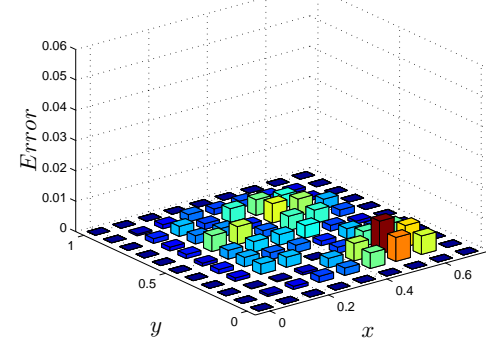

(c) Topology 3.

Fig. 9. Absolute value of the errors between the combined and reference global mode shapes (case 4, 2nd mode).

Repeating the simulation 100 times for each case, the averages of each error measure are calculated for the first seven modes as shown in Fig. 10 and Fig. 11. Zoomed figures from 0\% to 15\% are also provided in Fig. 11 to clearly show $E_{a v g, j}$. In Fig. 10 and Fig. 11, topology 3 consistently has the smallest errors in most cases. It can be concluded that sufficiently large local groups and multiple overlapping nodes as in Topology 3 contribute to reliable and accurate estimation of global modal properties.

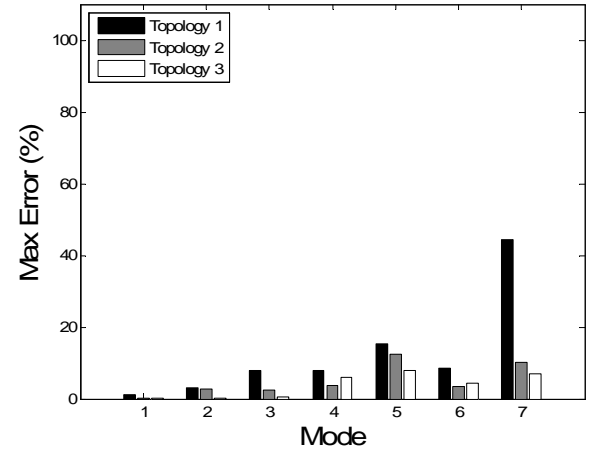

(a) Case 1: Impulse loading, input measured.

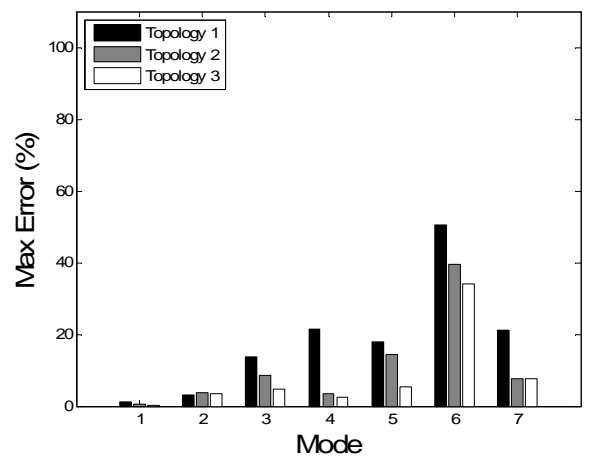

(c) Case 3: random excitation, input measured.

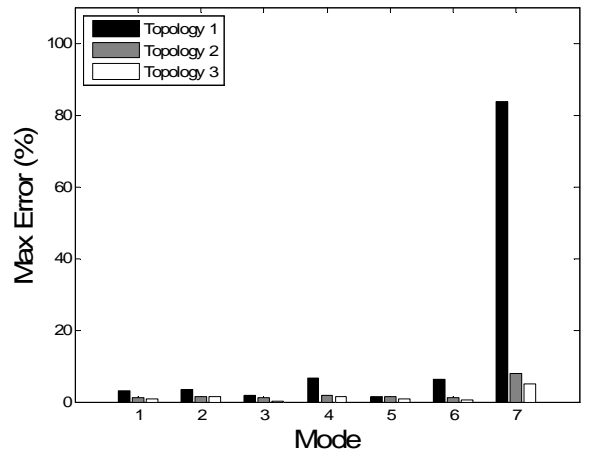

(b) Case 2: Impulse loading, input not measured.

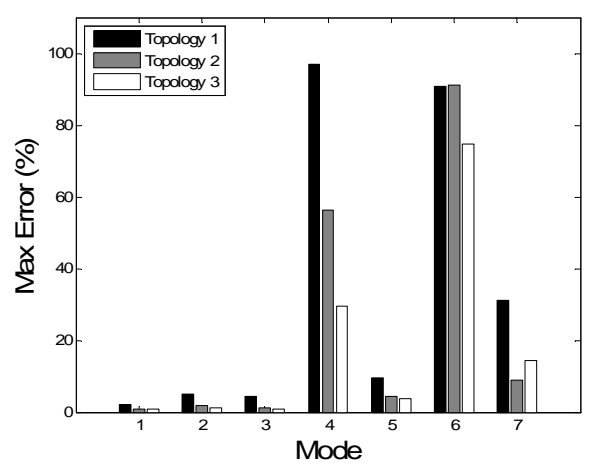

(d) Case 4: random excitation, input not measured.

Fig. 10. Maximum error. 


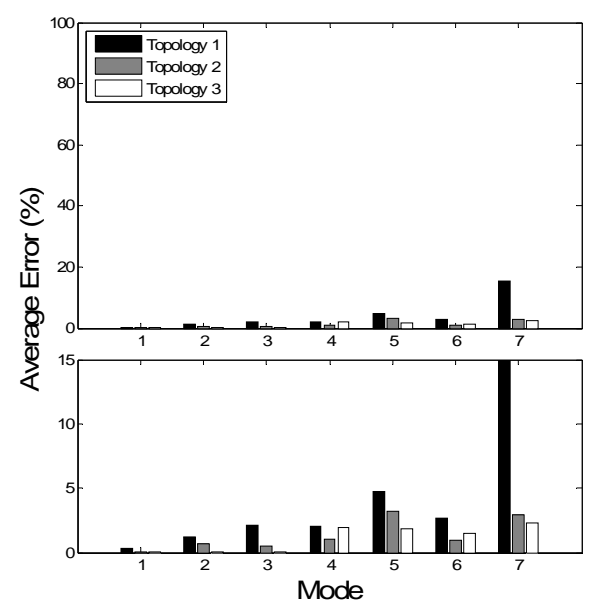

(a) Case 1: Impulse loading, input measured.

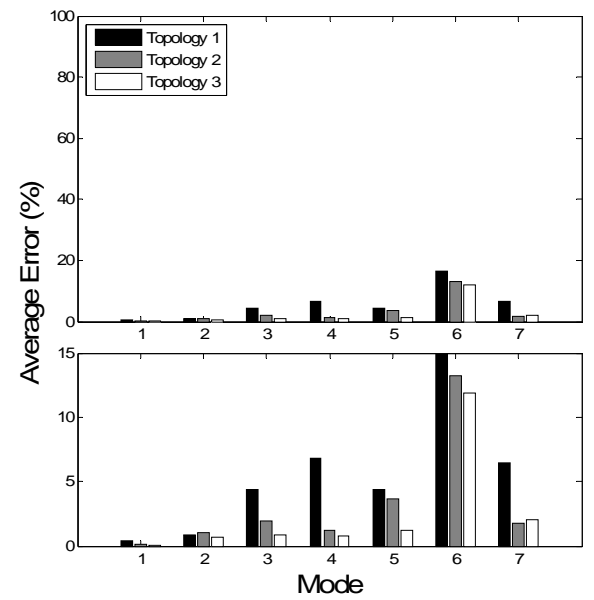

(c) Case 3: random excitation, input measured.

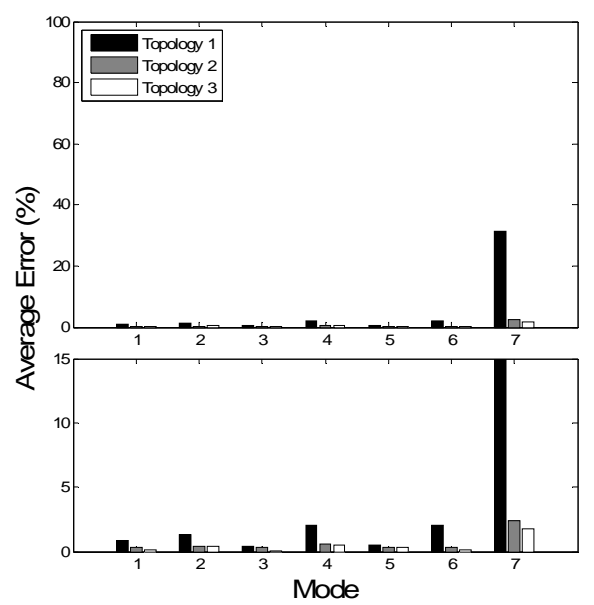

(b) Case 2: Impulse loading, input not measured.

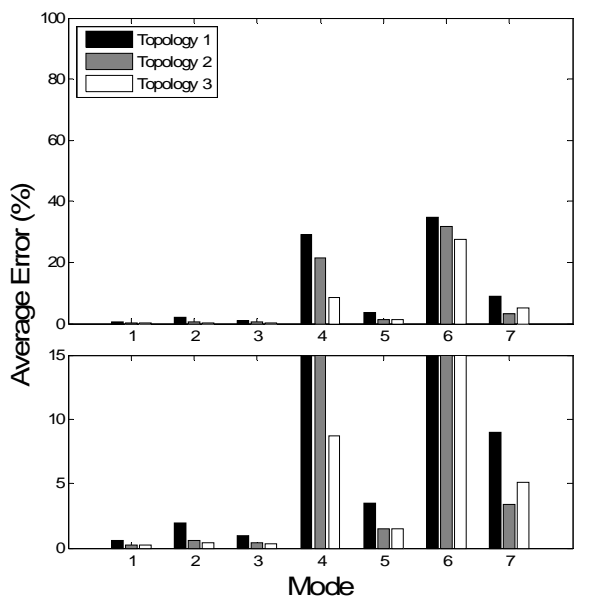

(d) Case 4: random excitation, input not measured.

Fig. 11. Average error.

\section{CONCLUSION}

An automated hierarchical decentralized approach for modal analysis using smart sensors has been proposed to obtain the global modal properties using a decentralized network topology. The proposed approach consists of two main parts: (1) local feature extraction and (2) determination of global modal properties. The modal analysis is conducted independently in each sensor group to estimate local information using ERA or NExT/ERA. Global modal properties are then obtained using the collected local properties in the base station. The number of local groups in which natural frequencies are locally identified is utilized to delineate between the true and noise modes. Then, local mode shapes are assembled using the least squares approximation to estimate the global mode shape.

The proposed approach was numerically validated. From the example of the plate, appropriately selected sensor topologies with the sufficiently large size of local groups and the multiple overlapping nodes were found to reduce errors in the assembled global mode shape. The numerical results show that the proposed approach for decentralized modal analysis is promising for the SSN. While ERA and NExT/ERA are used in this study to characterize the local modal 
properties, other system identification methods such as Stochastic Subspace Identification[19][20] or Frequency Domain Decomposition[21] can be alternatively used. Implementing the proposed approach on the SSN is currently underway.

\section{ACKNOWLEDGEMENT}

The authors gratefully acknowledge the support of this research by the National Science Foundation, under grant 0600433 (Dr. S. C. Liu, program manager) and Vodafone. The support is gratefully acknowledged.

\section{REFERENCES}

[1] Maia, N. M. M. and Silva, J. M. M., “Modal Analysis Identification Techniques,” Philosophical Transactions: Mathematical, Physical and Engineering Sciences 359(1778), 29-40 (2001).

[2] Dallard, P., Fitzpatrick, A. J., Flint, A., Le Bourva, S., Low, A., Ridsdill Smith, R. M., and Willford, M., "The London Millennium Footbridge,” The Structural Engineer 79(22), 17-33 (2001).

[3] Lynch, J. P., Sundararajan, A., Law, K. H., Kiremidjian, A. S., Carryer, E., Sohn, H., and Farrar, C., "Field Validation of a Wireless Structural Monitoring System on the Alamosa Canyon Bridge,” Proc. SPIE $10^{\text {th }}$ Annual Int. Symposium on Smart Structures and Materials, San Diego, CA, USA, March 2-6 (2003).

[4] Lynch, J. P., and Loh, K., "A summary review of wireless sensors and sensor networks for structural health monitoring,” Shock and Vibration Digest 38(2), 91-128 (2006).

[5] Tanner, N. A., Wait, J. R., Farrar, C. R., and Sohn, H., "Structural Health Monitoring Using Modular Wireless Sensors,” Journal of Intelligent Material Systems and Structures 14(1), 43-56 (2003).

[6] Caffrey, J., Govindan, R., Johnson, E., Krishnamachari, B., Masri, S., Sukhatme, G., Chintalapudi, K., Dantu, K., Rangwala, S., Sridharan, A., Xu, N., and Zuniga, M., "Networked Sensing for Structural Health Monitoring,” Proc. $4^{\text {th }}$ Int. Workshop on Structural Control, New York, NY, June 10-11, 57-66 (2004).

[7] Lynch, J. P., Sundararajan, A., Law, K. H., Kiremidjian, A. S., and Carryer,E., "Embedding Damage Detection Algorithms in a Wireless Sensing Unit for Operational Power Efficiency,” Smart Materials and Structures 13(4), 800-810 (2004).

[8] Lynch, J. P., Parra-Montesinos, G., Canbolat, B. A., and Hou, T-C., "Real-time Damage Prognosis of Highperformance Fiber Reinforced Cementitious Composite Structures,” Proc. Advances in Structural Engineering and Mechanics (ASEM’04), Seoul, Korea, September 2-4 (2004).

[9] Nitta, Y., Nagayama, T., Spencer Jr., B. F., and Nishitani, A., "Rapid damage assessment for the structures utilizing smart sensor MICA2 MOTE”, Proc $5^{\text {th }}$ Int. Workshop on Structural Health Monitoring, Stanford, CA., 283-290 (2005).

[10] Gao, Y., Spencer Jr., B. F. and Ruiz-Sandoval, M., "Distributed computing strategy for structural health monitoring," Journal of Structural Control and Health Monitoring 13(1), 488-507 (2006).

[11] Zimmerman, A. T., Shiraishi, M., Swartz, R. A., and Lynch, J. P., “Automated Modal Parameter Estimation by Parallel Processing within Wireless Monitoring Systems,” Journal of Infrastructure Systems 14(1), 102-113 (2008).

[12] Ewins, D. J., "Modal testing: Theory and practice,” Research Studies Press, Ltd., Staunton, Somerset, U.K. (1986).

[13] Allemang, R. J., "Vibrations: Experimental modal analysis," Course Notes (UC-SDRL-CN-20-263-663/664), Structural Dynamics Research Laboratory, University of Cincinnati, Cincinnati (http://www.sdrl.uc.edu/academiccourse-info/).

[14] Juang, J. N. and Pappa, R. S., “An Eigensystem realization algorithm for modal parameter identification and model reduction,” Journal of Guidance Control and Dynamics 8, 620-627 (1985).

[15] James, G. H., Carne, T. G., Lauffer, J. P. and Nord, A. R., "Modal testing using natural excitation,” Proc. $10^{\text {th }}$ Int. Modal Analysis Conference, San Diego, CA (1992).

[16] Nagayama, T. and Spencer Jr., B. F., "Structural Health Monitoring Using Smart Sensors,” Newmark Structural Engineering Laboratory (NSEL) Report Series, No. 1, University of Illinois at Urbana-Champaign, Urbana, Illinois (http://hdl.handle.net/2142/3521) (2007).

[17] Montgomery, D. C. and Runger, G. C., “Applied Statistics and Probability for Engineers,” John Wiley \& Sons, Inc. (1994).

[18] Zienkiewicz, O. C. and Cheung, Y. K., "The finite element method for analysis of elastic isotropic and orthotropic slabs,” Proc. Inst. Civ. Eng. 28, 471-488 (1964). 
[19] Van Overschee, P., and De Moor, B, “Subspace algorithms for the stochastic identification problem,” Automatica 29, 649-660 (1993).

[20] Hermans, L. and Van Der Auweraer, H., "Modal testing and analysis of structures under operational conditions: Industrial applications,” Mechanical Systems and Signal Processing 13(2), 193-216 (1999).

[21] Brincker, R., Zhang, L., and Andersen, P., "Modal identification of output-only systems using frequency domain decomposition,” Smart Materials and Structures 10, 441-445 (2001). 